nausea and vomiting lasting hours to days and separated by a return to baseline health.

The episodes are usually stereotypical in an individual patient, oftentimes preceded by prodromal symptoms. Similarly to other functional gastrointestinal disorders, the diagnosis of CVS relies on symptom-based Rome criteria IV. With the pathophysiology of the disorder still largely unknown, the management of CVS remains a challenge, especially in those with other comorbidities. Chiari malformation may manifest with recurrent vomiting, either due to raised intracranial pressure or to direct pressure on the brainstem.

Case Report We present a case of a female patient born with lumbosacral meningomyelocele and Chiari malformation type II whose attacks of nausea and vomiting started at the age of 10 months. The episodes were frequent, once to twice monthly, and stereotypical in their nature. Prior to vomiting, the child would suddenly become still and unresponsive, experiencing vegetative symptoms such as flushing and sneezing, followed by profuse bilious vomiting lasting up to a week. The girl's state usually required hospital admission and vigorous parenteral replenishment of fluid, electrolytes and energy.

Over the years, extensive diagnostic evaluation has been undertaken and no structural gastrointestinal nor metabolic abnormalities were found. Imaging studies, as well as neurological examination and diagnostic tests, did not help to elucidate the aetiology of recurring episodes. There were no signs of intracranial hypertension, VP drainage (performed in early childhood) was functional, repeated EEG did not reveal dysrhythmic changes. Treatment attempts including the sole administration of propranolol, amitriptyline, levetiracetam, ciproheptadine and topiramate and various combinations of said agents were unsuccessful in decreasing the frequency and severity of episodes. At the age of 7 , the patient underwent operative treatment of Chiari malformation type II. After craniocervical decompression, the episodes remained unchanged. Finally, aprepitant, NK-1 receptor antagonist, was introduced as a prophylactic and abortive agent when the girl was 8 years old. In the year following its introduction the episodes became much less frequent and shorter, never requiring parenteral hydration, leading to better somatic growth and improved health-related quality of life.

Conclusion Although there are no definitive treatments for CVS, NK-1 antagonist (aprepitant) may be effective as a prophylactic and/or abortive medication in refractory cases, leading to substantial improvement in the quality of life.

\section{MYCOPLASMA PNEUMONIAE INFECTIONS WITH ATYPICAL DEVELOPMENT IN CHILDREN - CASE PRESENTATION}

Nikola Pavlinović*, Ranka Despot, Sandra Prgomet. Public Health Centre of the SplitDalmatia County, Split, Croatia Department of Pediatrics, University Hospital of Split, Split, Croatia

\subsection{6/archdischild-2021-europaediatrics.254}

Introduction Lower respiratory tract infections are considered a common cause responsible for morbidity and mortality among children, and Mycoplasma pneumoniae is identified to be responsible for up to 40 per cent of community-acquired pneumonia in children greater than five years of age [1] and also in $20 \%$ of adult cases [2].

Extrapulmonary manifestations have been reported either due to spread of infection or autoimmune mechanisms [1]. A case report on necrotizing pancreatitis was issued by Yang et al., 2015 [3].

Case 1. A 9-year old boy was admitted to our hospital presenting with an acute febrile illness lasting for four days associated with a generalised, centripetal rash and macrohaematuria. He had been previously treated with azythromycin for three days. Past medical history revealed that tonsillectomy was performed at 5 years of age and the diagnosis of hypoacusis perceptiva was made. On initial assessment he apeared well, alert and conscious. He was subfebrile $\left(37.6^{\circ} \mathrm{C}\right)$. with a maculopapular confluented rash on the trunk and proximal parts of lower limbs. Initial investigation revealed elevated sedimentation rate $(54 \mathrm{~mm} / \mathrm{h})$, leukocytosis $\left(16,7 \times 10^{\wedge} 9 / \mathrm{L}\right)$, normal hemoglobin level and normal total red cell count. The patient had slightly elevated bilirubin (total bilirubin 65.8 , conjugated $44.6 \mu \mathrm{mol} / \mathrm{L}$ ), elevated AST (342 U/ L), ALT (345 U/L), and GGT (534 U/L). His renal function and electrolyte panel was normal. Chest X-ray was normal without any lesions in the lungs. Urine investigation revealed macrohematuria, proteinuria with active urinary sediment (dismorfic erythrocytes and erythrocyte casts). On ultrasound kidneys were enlarged with hyperechogenic parenchyma, diminished corticomedular differentiation. Because of proteinuria (total protein $2829 \mathrm{mg} / 24$ hours, albumin $1394 \mathrm{mg} / 24$ hours), and hematuria, kidney biopsy was performed. On light microscopy we found mesangihypercellurality, interstitial fibrosis and tubular atrophy (focal). On IF microscopy there was a poorly expressed granular deposit of $\operatorname{IgM}$ on the glomerular basement membrane (GBM) with no IgA, IgG, C1q, C3 and C4 immune deposits. On electron microscopy the GBM was of variable width (113 to $670 \mathrm{~nm}$, average $303 \mathrm{~nm}$, SD 164). In the thicker part of GBM lamelation was present. Podocytes were normal. The pathohystologic exam was consistent with Alport syndrome. Mycoplasma serology was consistent with acute infection, with Mycoplasma IgM positive $(26.3 \mathrm{U} / \mathrm{mL})$, and negative IgG.

Case 2. A 15-year old male adolescent was admitted to hospital with symptoms of abdominal pain lasting for two weeks, with no nausea or vomiting, and normal stool passing. The boy was living with his mother, who had been diagnosed with neurofibromatosis, in an atypical family situation of divorced parents.

Physical examination showed abdominal pain in the left upper quadrant, also spreading to the back and lumbar region. Initial laboratory analysis showed a slight increase in serum amylase $(140 \mathrm{U} / \mathrm{L})$ and lipase $(518 \mathrm{U} / \mathrm{L})$. The C-reactive protein was inside referent range $(2 \mathrm{mg} / \mathrm{L})$ as were the value of liver enzymes (AST, ALT, GGT). The TSH was inside referent range, and the antibodies related to gluten enteropathy came negative (anti-tTg-IgA and anti-DGP-IgG). The serology results showed positive antibodies to Mycoplasma pneumoniae (IgM positive, $60.3 \mathrm{U} / \mathrm{ml}$, IgG positive, $25.8 \mathrm{U} / \mathrm{ml}$ ). We also proved positive IgG antibodies to parvovirus B19, cytomegalovirus, and herpes simplex virus (HSV 1/2). MRI of the abdomen showed a focal pancreatitis (changed signal of the pancreas tail parenchyma).

The patient received standard treatment (pancreatic diet and proton-pump inhibitor) and an improvement was perceived with a slow decrease of serum amylase and lipase. Due to a later onset of fever with respiratory symptoms, clarithromycin therapy was initiated. This patient had a second episode of acute pancreatitis nine months after the described event with somewhat larger initial values of serum amylase (154 U/L) and lipase (1533 U/L), and also elevated urine 
sample values. The patient had reccurent problems with social functioning, which manifested as intermittent palpitations, precordial sensations, and a prolonged episode of large amplitude incontrollable tremor for which he was hospitalized once again after the second episode of pancreatitis.

Conclusion The presented cases show the possible extent of extrapulmonary illness caused by Mycoplasma pneumoniae in children. Judging by these cases, autoimmune mechanisms and also genetic factors seem to play an important role in the infection of a specific organ by $\mathrm{M}$. pneumoniae.

\section{REFEEDING SYNDROME IN HOSPITALIZED CHILDREN AND ADOLESCENTS WITH EATING DISORDERS- EXPERIENCE OF TERTIARY CENTER}

Barbara Perše*, Ana Kovačević, Andrijana Kološa, Matea Crnković Ćuk, Bruno Špiljak, Luka Šimunović, Orjena Žaja. Sestre Milosrdnice University Hospital Center (Zagreb, Croatia)

10.1136/archdischild-2021-europaediatrics. 255

Refeeding syndrome (RFS) describes potentially fatal shift in electrolytes in severe malnourished patients receiving rapid and excessive food re-introduction. It is a result of hormonal and metabolic disturbances.

There are various clinical and laboratory features with hypophosphatemia being the most common one. Elevation in liver function tests is also frequently seen. Patients with anorexia are a high-risk group for developing RFS. The aim of the study was to investigate the incidence and clinical features of RFS among hospitalized patients, as well as severity of malnutrition (Z-score, BMI).

This study is a retrospective analysis of medical documentation of patients diagnosed with anorexia nervosa (restricting (ANRT) and binge eating/purging

(ANBP) subtype), eating disorder NOS (EDNOS) and avoidant/restrictive food intake disorder (ARFID) who were admitted to our Centre for eating disorders in children and adolescents during a 5 year period (2014-2018). We analyzed the age, gender, duration of the disease before admittance, anthropometric data (BMI and Z-score), average weight loss, the need for nasogastric (NG) tube feeding and phosphate supplementation. For statistical analysis we used t-test.

256 patients (232 female) aged 6-20 years (median 15+/2.06 ) of which $43 \%$ were diagnosed with ANRT, 10\% ANBP, 8\% ARFID, 39\% with EDNOS were included in the study. The average duration of the disease at the time of admittance was 13.25+/-13.43 months. Average BMI Z-score was -1.97 $+/-1.63$ average weight loss was $20+/-9.76 \%$ initial body weight (IBW).

Hypophosphatemia was found in $15.6 \%$ patients, of which $65 \%$ received phosphate supplements by oral or intravenous route depending of phosphate serum concentration. Elevated liver enzymes due to RFS were found in $9.3 \%$ of patients. In total RFS in some form developed in 23\% patients.

Average BMI Z-score of patients that developed RFS was $2.6+/-1.89$, average weight loss was $23.9+/-9.85 \%$ of IBW. Both variables were significantly different $(p<0.05)$ in comparison with non-RFS group which had BMI Z-score of

$-1.76+/-1.47$ and average weight loss of $18.7+/-9.3 \%$ of IBW. The average duration of the disease was similar in both groups (13 months) $(\mathrm{p}=0.84)$.
NG tube feeding was needed in $27 \%$ of all patients, $24 \%$ patients in non-RFS group and $37 \%$ in patients with RFS.

Our study reported that even in controlled hospital conditions and with careful realimentation RFS has a high incidence. We found statistically significant difference when it comes to BMI Z-score and average weight loss between two groups of patients.

\section{HEMOPHAGOCYTIC LYMPHOHISTIOCYTOSIS IN A 13- YEAR OLD GIRL WITH CROHN'S DISEASE}

Barbara Perše*, Marina Mataija, Matej Katavić, Ika Kardum-Skelin, Matea Crnković Ćuk, Orjena Žaja. Sestre Milosrdnice University Hospital Center

\subsection{6/archdischild-2021-europaediatrics.256}

Hemophagocytic lymphohistiocytosis (HLH) is a severe, lifethreatening disorder of uncontrolled immune activation and inflammation which, if left untreated, can lead to organ failure and death. Mortality is high, so prompt recognition and treatment are crucial. However, clinical presentation is unspecific, condition is rare, so in some cases the diagnosis and treatment can be delayed, which further impairs chances of recovery. In recent years, the number of reported cases of HLH in inflammatory bowel disease (IBD) patients has increased, probably due to higher awareness than real rise in incidence, although this condition is still often overlooked.

We describe a 13-year-old girl with Crohn's disease (CD) on azathioprine therapy, who developed HLH as a complication of primary CMV infection and EBV reactivation.

13-year-old girl was diagnosed with $\mathrm{CD}$ two years prior and was in stable remission with standard pediatric therapy, enteral therapy, followed by azathioprine maintenance therapy, which she was currently taking. The function of TPMT was normal.

Two months after last follow up she was admitted in poor clinical condition with a prolonged period of high-grade fever, fatigue, poor appetite, weight loss, few episodes of vomiting associated with peaks of fever and slight abdominal tenderness. No other symptoms suggestive of CD relapse were present which was later confirmed by normal ileocolonoscopy and MR enterography. According to history she was in contact with infectious mononucleosis patient two months prior. Azathioprine was discontinued. After obtaining specimens for microbiological analysis empirical broad-spectrum antibiotics were initiated. During next few days her clinical state started to deteriorate. At this point, additional tests confirmed our suspicion of

HLH: persistent fever, bicytopenia, hyperferritinemia, hypofibrinogenemia, hypertriglyceridemia, low NK activity, splenomegaly, and CD68 and ANA positive phagocytes in bone marrow smear. All bacterial cultures came back negative. Serology and PCR results revealed that HLH was a complication of primary CMV infection with EBV reactivation.

Treatment with systemic corticosteroids was initiated with excellent response, lithic drop of fever, improvement in general condition and normalization of biochemical markers. The dose of corticosteroids was tapered gradually and her clinical state was monitored closely. She had no signs of relapses of HLH, primary disease, nor no signs of malignant disease during one year of follow-up period. 